\title{
Diffusion tensor imaging on 3-T MRI breast: diagnostic performance in comparison to diffusion-weighted imaging
}

Dalia Abdelhady ${ }^{1,2^{*}}$ (D), Amany Abdelbary ${ }^{3}$, Ahmed H. Afifi', Alaa-eldin Abdelhamid ${ }^{1}$ and Hebatallah H. M. Hassan ${ }^{1}$

\begin{abstract}
Background: Breast cancer is the most prevalent cancer among females. Dynamic contrast-enhanced MRI (DCEMRI) breast is highly sensitive (90\%) in the detection of breast cancer. Despite its high sensitivity in detecting breast cancer, its specificity (72\%) is moderate. Owing to 3-T breast MRI which has the advantage of a higher signal to noise ratio and shorter scanning time rather than the 1.5-T MRl, the adding of new techniques as diffusion tensor imaging (DTI) to breast MRI became more feasible.

Diffusion-weighted imaging (DWI) which tracks the diffusion of the tissue water molecule as well as providing data about the integrity of the cell membrane has been used as a valuable additional tool of DCE-MRI to increase its specificity.

Based on DWI, more details about the microstructure could be detected using diffusion tensor imaging. The DTI applies diffusion in many directions so apparent diffusion coefficient (ADC) will vary according to the measured direction raising its sensitivity to microstructure elements and cellular density. This study aimed to investigate the diagnostic accuracy of DTI in the assessment of breast lesions in comparison to DWI.

Results: By analyzing the data of the 50 cases (31 malignant cases and 19 benign cases), the sensitivity and specificity of DWI in differentiation between benign and malignant lesions were about $90 \%$ and $63 \%$ respectively with PPV 90\% and NPV 62\%, while the DTI showed lower sensitivity and specificity about $81 \%$ and $51.7 \%$, respectively, with PPV 78.9\% and NPV 54.8\% ( $P$-value $\leq 0.05$ ).
\end{abstract}

Conclusion: While the DWI is still the most established diffusion parameter, DTI may be helpful in the further characterization of tumor microstructure and differentiation between benign and malignant breast lesions.

Keywords: Diffusion tensor magnetic resonance imaging, Diffusion-weighted MRI, Fractional anisotropy, 3 Tesla MRI breast, Cancer breast

\footnotetext{
* Correspondence: Daliahady204@gmail.com

'Department of Diagnostic and Interventional Radiology, Faculty of Medicine, Alexandria University, Alexandria, Egypt

${ }^{2}$ Department of Diagnostic and Interventional Radiology, Faculty of Medicine, Alexandria University Hospital, Champollion Street, Al-Azarita,

Alexandria 21526, Egypt

Full list of author information is available at the end of the article
}

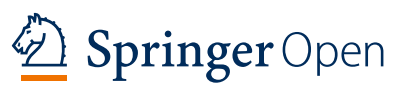

(c) The Author(s). 2021 Open Access This article is licensed under a Creative Commons Attribution 4.0 International License, which permits use, sharing, adaptation, distribution and reproduction in any medium or format, as long as you give appropriate credit to the original author(s) and the source, provide a link to the Creative Commons licence, and indicate if changes were made. The images or other third party material in this article are included in the article's Creative Commons licence, unless indicated otherwise in a credit line to the material. If material is not included in the article's Creative Commons licence and your intended use is not permitted by statutory regulation or exceeds the permitted use, you will need to obtain permission directly from the copyright holder. To view a copy of this licence, visit http://creativecommons.org/licenses/by/4.0/. 


\section{Background}

Worldwide, breast cancer is the most prevalent cancer among females and is estimated as the second cause of cancer-related mortality [1].

In the diagnosis of breast cancer, mammography and ultrasound are proven imaging modalities [2], but in some cases, these imaging approaches can lead to inconclusive results where breast cancer cannot be excluded. Here, breast MR imaging can be used as a problem-solving technique to avoid or guide biopsies owing to its high sensitivity and soft-tissue contrast [3].

Breast MRI is a highly sensitive imaging method for the detection of breast cancer $[4,5]$, in particular when the breast tissue on the mammogram is dense [6, 7]. It has also been accepted for other clinical indications, such as screening in high risk females and breast cancer assessment prior to surgical intervention [8].

The 3-T MR imaging has the advantage of a higher signal to noise ratio, better spatial resolution, and shorter scanning time rather than the 1.5-T MRI leading to better characterization of breast lesions [9].

Owing to 3-T breast MRI, the path for more advanced modalities, including spectroscopic imaging, bloodoxygen-level-dependent (BOLD) contrast imaging, and diffusion tensor imaging (DTI) of the breast is paved and became more feasible [10].

Despite the high sensitivity of dynamic contrastenhanced (DCE)-MRI in detection of breast cancer (90\%), its specificity $(72 \%)$ is moderate $[2,11]$.

Diffusion-weighted imaging (DWI), which tracks the diffusion of the tissue water molecule as well as providing data on the integrity of the cell membrane, has been used as a valuable additional tool of dynamic contrastenhanced MRI (DCE-MRI) to increase its specificity and decrease unnecessary biopsies. Currently, the DWI is commonly utilized to identify, characterize, and discriminate malignant and benign lesions $[2,12]$.

Table 1 Distribution of the studied cases according to BIRADS based on mammogram and/or ultrasound ( $N=50)$

\begin{tabular}{lll}
\hline BIRADS mammogram and ultrasound & No. & $\%$ \\
\hline 0 & 1 & 2.0 \\
1 & 1 & 2.0 \\
2 & 4 & 8.0 \\
3 & 12 & 24.0 \\
4 & 18 & 36.0 \\
$4 a$ & 7 & 14.0 \\
$4 b$ & 4 & 8.0 \\
$4 c$ & 7 & 14.0 \\
5 & 12 & 24.0 \\
6 & 2 & 4.0 \\
\hline
\end{tabular}

Based on DWI, more details about the microstructure could be detected using diffusion tensor imaging (DTI) [13]. DTI could be considered a more detailed form of DWI $[14,15]$.

The breast is characterized by unique morphology. It is composed of a mixture of fatty and fibroglandular components. Fibroglandular tissue is divided into several lobes [16].

In the mammary ducts and lobules, the diffusion of water molecules tends to be restricted and anisotropic. The parallel movement of water molecules to the walls of the duct and lobules leads to free diffusion [17-19], while the vertical movement of water molecules to the walls of cells and basement membrane, on the contrary, results in restricted diffusion [17-19].

In case of malignancy, the mammary ducts and lobules are blocked by the malignant cells leading to restricted water diffusion [17-19].

In the DWI sequence, apparent diffusion coefficient (ADC) assess the movement of water molecules according to Brownian motion regardless to the direction of movement. On the other hand, the DTI applies diffusion in many directions so ADC will vary according to the measured direction raising its sensitivity to microstructural elements and cellular density.

Considering the differences in ADC in different direction, fraction anisotropy (FA) provides more information about the diffusion of water molecules the three main $x-, y$-, and $z$-axes [20].

This presented study aimed to investigate the diagnostic accuracy of DTI in assessing breast lesions in comparison to DWI and to investigate the value of adding DTI as an adjunct to the standard breast imaging protocol using a 3-T MRI machine.

\section{Methods}

A prospective observational study was done by enrollment of 50 female patients referred to the MRI unit of the Radio-diagnosis Department at our university hospital during the time interval from June 2018 to January 2020.

The included patients were (a) female patients aged 25-70 years old, (b) presented with breast or axillary complain confirmed by clinical examination and followed by further assessment by mammogram and/or ultrasound breast, and (c) all Breast Imaging and Reporting System (BIRADS) categories were included from BIRADS 0 to BIRADS 6 to investigate both benign and malignant breast lesions.

Exclusion criteria were (a) breast lesion in male patient, (b) patients with primary tumor other than breast cancer to ensure assessment of primary breast tumors, and (c) patients receiving neoadjuvant chemotherapy or recent breast radiotherapy and patients with prior reduction mammoplasty or augmentation to avoid 
Table 2 Distribution of the studied cases according to CE-MRI findings $(N=50)$

\begin{tabular}{llll}
\hline MRI findings & & & \\
\hline & $N=50$ & & \\
\hline $\begin{array}{l}\text { Background } \\
\text { enhancement }\end{array}$ & & & \\
Minimal & 22 & 44.0 & \\
Mild & 14 & 28.0 & \\
Moderate & 11 & 6.0 & \\
Marked & 3 & & \\
& $\boldsymbol{N}=\mathbf{5 0}$ & $\mathbf{6 8 . 0}$
\end{tabular}

N. of cases $(N=34) \quad n$. of masses $(n=84)$ Shape

$\begin{array}{llll}\text { Oval } & 11 & & 18 \\ \text { Round } & 2 & & 8 \\ \text { Irregular } & 21 & & 58 \\ & & \text { Margins } & \end{array}$

Circumscribed $\quad 13 \quad 26$

Irregular $\quad 10 \quad 26$

Speculated

11

32

$\begin{array}{lll}\text { Homogenous } & 13 & 26 \\ \text { Heterogeneous } & 17 & 43 \\ \text { Dark internal } & 4 & 15\end{array}$

septations

$$
N=50
$$

Non-mass enhancement

\begin{tabular}{|c|c|c|}
\hline No & 24 & 48.0 \\
\hline Yes & 26 & 52.0 \\
\hline Morphology & $N=26$ & \\
\hline Clumped & 21 & 80.8 \\
\hline Homogenous & 4 & 15.4 \\
\hline Clustered ring & 1 & 3.8 \\
\hline Distribution & $N=26$ & \\
\hline Segmental & 12 & 46.0 \\
\hline Regional & 3 & 11.5 \\
\hline Linear & 9 & 34.5 \\
\hline Focal & 1 & 4.0 \\
\hline Diffuse & 1 & 4.0 \\
\hline
\end{tabular}

Dynamic curve

$$
\begin{aligned}
& \text { Type I } \\
& \text { Type ॥ } \\
& \text { Type II }
\end{aligned}
$$

Pectoralis muscle

\begin{tabular}{|c|c|c|}
\hline \multicolumn{3}{|l|}{ MRI findings } \\
\hline \multicolumn{3}{|c|}{$N=50$} \\
\hline \multicolumn{3}{|c|}{ Axillary lymph nodes } \\
\hline Reactive looking & 29 & 58.0 \\
\hline Malignant looking & 17 & 34.0 \\
\hline Equivocal & 4 & 8.0 \\
\hline \multicolumn{3}{|c|}{ BIRADS } \\
\hline 1 & 0 & 0.0 \\
\hline 2 & 5 & 10.0 \\
\hline 3 & 11 & 22.0 \\
\hline 4 & 10 & 20.0 \\
\hline $4 a$ & 3 & 6.0 \\
\hline $4 b$ & 2 & 4.0 \\
\hline $4 c$ & 5 & 10.0 \\
\hline 5 & 8 & 16.0 \\
\hline 6 & 16 & 32.0 \\
\hline
\end{tabular}
invasion
Table 2 Distribution of the studied cases according to CE-MRI findings ( $N=50)$ (Continued)

the related effect on DWI and DTI values, in addition to the general contraindication of MRI including patients with severe medical illness as renal failure.

After thorough history taking about patient's complaint, age, and risk factors for breast cancer, clinical examination of bilateral breast, and axillae, the radiological assessment was performed.

Combined diagnostic mammogram and ultrasound breast were done in 44 cases out of 50 , which were 40 years or older, apart from one case aged 35 years old with 1st degree positive family history of cancer breast presented with breast lump, while the remaining 6 cases underwent ultrasound breast only due to age less than 40. CE-MRI breast including DWI with added DTI sequence were done to the all fifty cases.

CE-MRI breast was performed on a 3-T MRI machine (Ingenia Philips-Netherland, 2011), using a dedicated

Table 3 Distribution of the studied cases according to biopsy $(N=50)$

\begin{tabular}{lll}
\hline Biopsy & No. & \% \\
\hline No biopsy & 8 & 16.0 \\
Biopsied cases & $\mathbf{4 2}$ & $\mathbf{8 4 . 0}$ \\
Fine needle cytology & 19 & \\
Tru cut biopsy (TCNB) & 27 & \\
Excisional biopsy & 6 & \\
Nipple discharge cytology & 5 & \\
\hline Some lesions were biopsied by more than one type of biopsy
\end{tabular}


Table 4 Distribution of the biopsied cases according to histopathology results $(N=42)$

\begin{tabular}{lll}
\hline Histopathology & No. & \% \\
\hline Benign & $\mathbf{1 1}$ & $\mathbf{2 6 . 3}$ \\
Fibroadenoma & 4 & 9.5 \\
Fibroadenosis with or without atypia & 2 & 4.8 \\
Duct papillomatosis & 2 & 4.8 \\
Granulomatous mastitis & 1 & 2.4 \\
Fat necrosis & 1 & 2.4 \\
Lymphocytic mastitis & 1 & 2.4 \\
Malignant & $\mathbf{3 1}$ & $\mathbf{7 3 . 7}$ \\
IDC & 20 & 47.6 \\
DCIS & 7 & 16.7 \\
Lobular carcinoma & 2 & 4.7 \\
Mucinous carcinoma & 1 & 2.4 \\
Paget's disease of nipple & 1 & 2.4 \\
\hline
\end{tabular}

phased array 16 channel breast coil. The examination was scheduled in the 2nd week of the menstrual cycle in premenopausal women. In cases referred with BIRADS 6 classification, the MRI was done 10-14 days after the biopsy to eliminate effect of edema or hematoma.

1. Routine sequences of MRI breast: T2-weighted, fat-saturated sequence (STIR) with TR (5000), TE (60), slice thickness $2 \mathrm{~mm}$, and scanning time about 5 min. T1-weighted non-fat-saturated sequence with TR (shortest), TE (4.7), FOV (270$340 \mathrm{~mm})$, matrix $(448 \times 323)$, slice thickness $(1$ $\mathrm{mm})$, and gap $(20 \%=0.8 \mathrm{~mm})$ in axial plane with extension to the axillae, scan time about 5 min. Diffusion-weighted echo-planar imaging (EPI) sequence before contrast media injection with TR (shortest), TE (95 ms), FOV (270-340 mm), matrix (192x192), slice thickness $(4 \mathrm{~mm})$, and gap $(50 \%=2 \mathrm{~mm})$ in an axial plane. The $b$ values are 0,400 , and 800 with computergenerated ADC map, scan time 5-6 min. 3D T1weighted fat suppression gradient echo sequence performed before and repeated five times after IV administration of $0.2 \mathrm{mmol}$ gadolinium chelate $/ \mathrm{kg}$ at a rate of $3-4 \mathrm{~mL} / \mathrm{s}$, followed by $20 \mathrm{~mL}$ saline flush. The repeated sequences are taken with 60$\mathrm{s}$ intervals. TR and TE shortest, slice thickness 1 $\mathrm{mm}$, and fat suppression is spectral attenuated inversion recovery (SPAIR), scan time 8-9 min.

2. Diffusion tensor sequence (DTI)

(a) It was acquired before contrast administrations due to probable adverse effects on the signal in ssEPI (single-shot spin echo-prepared echoplanar imaging).

(b) Axial 2D diffusion-weighted ssEPI sequence with fat suppression (SPAIR) with the following parameters: TR $6000 \mathrm{~ms}$, TE $65 \mathrm{~ms}$; matrix, 240 $\times 240$; FOV $360 \times 360 \mathrm{~mm}$; in-plane voxel size, slice thickness $3 \mathrm{~mm}$; and gap, 0. Diffusion gradients were applied in fifteen directions with three $b$ values of 0,400 , and $800 \mathrm{~s} / \mathrm{mm}^{2}$.

(c) Number of diffusion gradient direction (NDGD): We used the 15-NDGD protocol (medium number of direction) to obtain an accurate fraction anisotropy map (more accurate than low number of direction $=6$ ) and to decrease the time of acquisition down to 5-10 min for the sequence (shorter time than high direction $=32$, which reaches up to 15 min)

(d) Computer-generated FA map

(e) The whole MRI study about 25-35 min

Table 5 Distribution of the studied cases according to FA and ADC in correlation to final diagnosis ( $N=50)$

\begin{tabular}{|c|c|c|c|c|c|}
\hline FA values & \multicolumn{2}{|c|}{$N=50(n=91)$} & ADC values & \multicolumn{2}{|c|}{$N=50(n=91)$} \\
\hline Min-max & \multicolumn{2}{|c|}{$0.05-0.48$} & Min-max & \multicolumn{2}{|c|}{$0.50-2.40$} \\
\hline Mean \pm SD & \multicolumn{2}{|c|}{$0.26 \pm 0.096$} & Mean \pm SD & \multicolumn{2}{|c|}{$1.21 \pm 0.45$} \\
\hline Median (IQR) & \multicolumn{2}{|c|}{$0.27(0.18-0.32)$} & Median (IQR) & \multicolumn{2}{|c|}{$1.15(0.80-1.50)$} \\
\hline Correlation between FA and final diagnosis & $N=50$ & $\%$ & Correlation between ADC and final diagnosis & $N=50$ & $\%$ \\
\hline Matching & 30 & 60 & Matching & 37 & 74 \\
\hline Borderline & 4 & 8 & Borderline & 4 & 8 \\
\hline Not Matching & 16 & 32 & Not Matching & 9 & 18 \\
\hline FA matching values with final diagnosis & $N=30$ & $\%$ & ADC matching values with final diagnosis & $N=37$ & $\%$ \\
\hline Malignant cases & 22 & 73 & Malignant cases & 20 & 54 \\
\hline Benign cases & 8 & 27 & Benign cases & 17 & 46 \\
\hline
\end{tabular}

$N$ number of cases, $n$ number of lesions. 


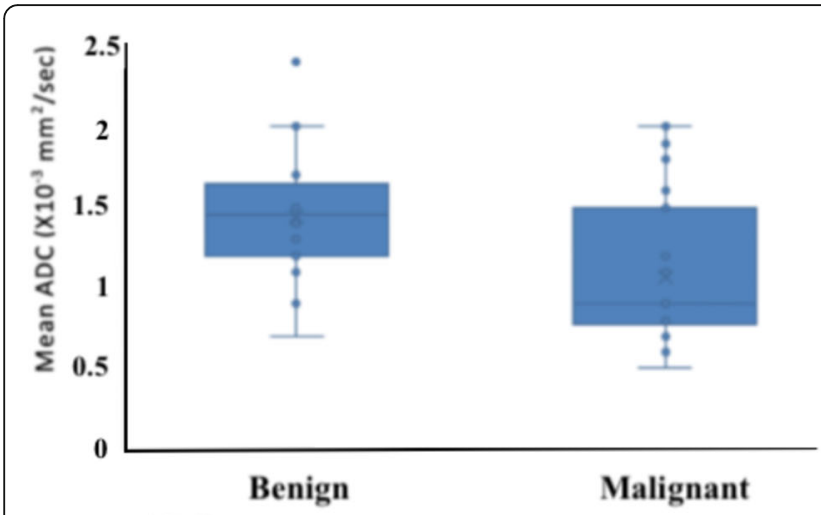

ADC

Fig. 1 Boxplot of ADC and FA values according to lesion's pathology (benign and malignant lesions)

Image post-processing and reading were performed using an extended MR workstation (IntelliSpace Portal) by two qualified radiologists, with 13 and 5 years of experience who were blinded from histopathological findings. The processing was done by each radiologist independently and the inter-observer agreement was nearly perfect agreement (kappa = 0.87; 95\% CI 0.60-0.99). Dual discussion was made after the separate evaluation and a consensus was reached to resolve any minor discrepancies for the final results.

The fifty cases showed total number of 98 breast lesions, and some lesions were excluded from the study; when DWI or DTI showed sub-optimal images due to artifacts $(n=5)$ and lesions which were not adequately assessed in dynamic contrast images due to failed fat saturation sequel to technical problems $(n=2)$. Total final number of the studied lesions were $(n=91)$.

In DWI and DIT, multiple ROIs were used. ROI site was based on the voxels that showed the most enhancement. That was correlated with voxels that showed a maximum restriction values at high $b$ value $\left(800 \mathrm{~s} / \mathrm{mm}^{2}\right)$.

In a trial to achieve standardized conditions for results analysis and avoid data contamination by adjacent structures, multiple elliptical regions of interest (ROI), with a mean area of $30 \mathrm{~mm}^{2}$ (ranging from 10 to $50 \mathrm{~mm}^{2}$ ), were individually placed on the ADC

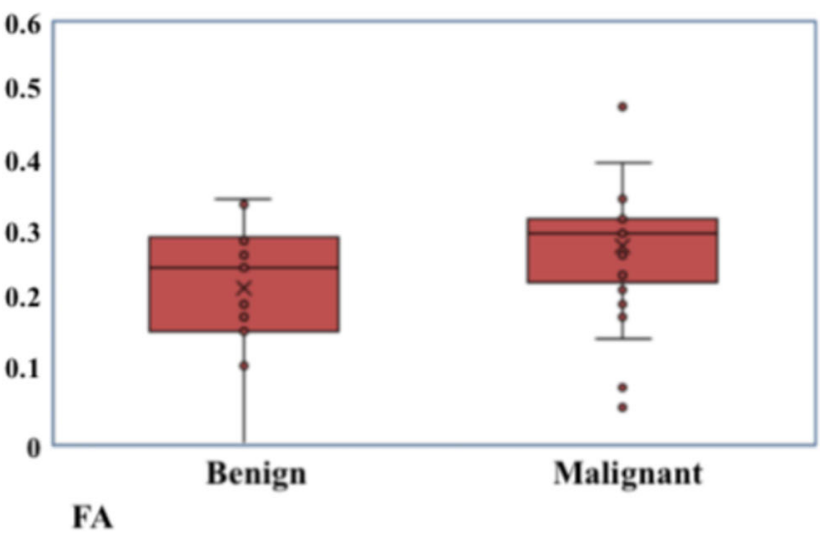

FA and FA maps at the site of the target lesion and mean ADC and FA were calculated. Necrotic or cystic components were avoided.

Cut-off values of ADC and FA for differentiation between benign and malignant lesions were calculated according to histopathological results using ROC (receiver operating characteristic) curve.

In cases with multiple detected lesions either unilateral or bilateral, the mean FA and mean ADC of the lesions were calculated as final values of the case and overall BIRADS assessment was according to ACR recommendation in latest BIRADS edition (2013); based on the most abnormal lesion and the highest likelihood of malignancy of the two breasts.

Gold standard assessment of the diagnostic outcome of DWI and DTI of 3-T CE-MRI was based upon the following:

- Histopathological correlation in indicated cases for biopsy based upon clinical and imaging findings and post-operative data whenever surgical excision was done $(n=42)$.

- Clinical and ultrasound imaging follow-up after 6 months for 8 cases classified as BIRADS 2 or 3 .

Followed by statistical analysis of the data, data were fed to the computer and analyzed using IBM SPSS software package version 20.0. (Armonk, NY:

Table 6 Diagnostic performance (sensitivity, specificity) of ADC and FA to predict breast malignant tumor (vs. benign)

\begin{tabular}{llllllllll}
\hline & AUC & $P$-value & $\mathbf{9 5 \%}$ Cl & Cut-off & Sensitivity & Specificity & PPV & NPV & Accuracy \\
\hline ADC & $74.2 \%$ & $0.004^{*}$ & $(0.885,0.60)$ & $1 \times 10^{-3} \mathrm{~mm}^{2} / \mathrm{s}$ & $90 \%$ & $63 \%$ & $90 \%$ & $62 \%$ & $53 \%$ \\
FA & $67.7 \%$ & $0.035^{*}$ & $(0.524,0.829)$ & 0.2 & $81 \%$ & $51.7 \%$ & $78.9 \%$ & $54.8 \%$ & $33 \%$ \\
\hline
\end{tabular}

$A U C$ area under a curve, $P$-value probability value, $P P V$ positive predictive value, NPV negative predictive value

* Statistically significant at $p \leq 0.05$ 


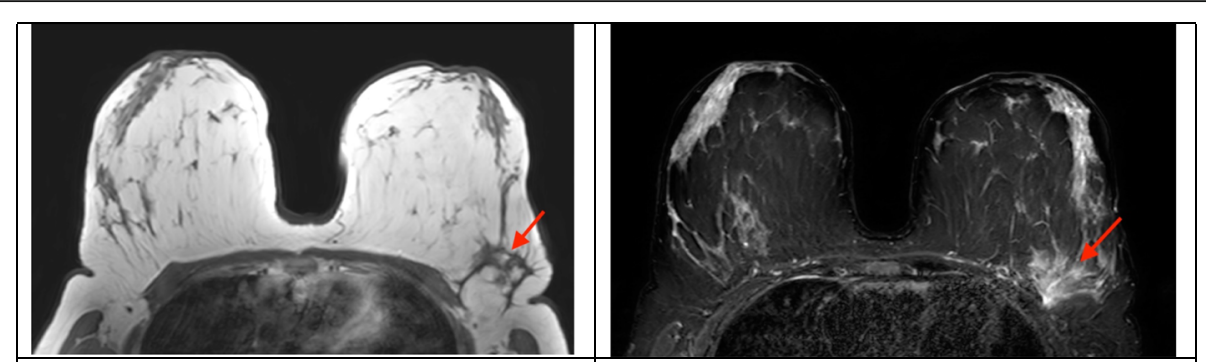

(a)

(b)

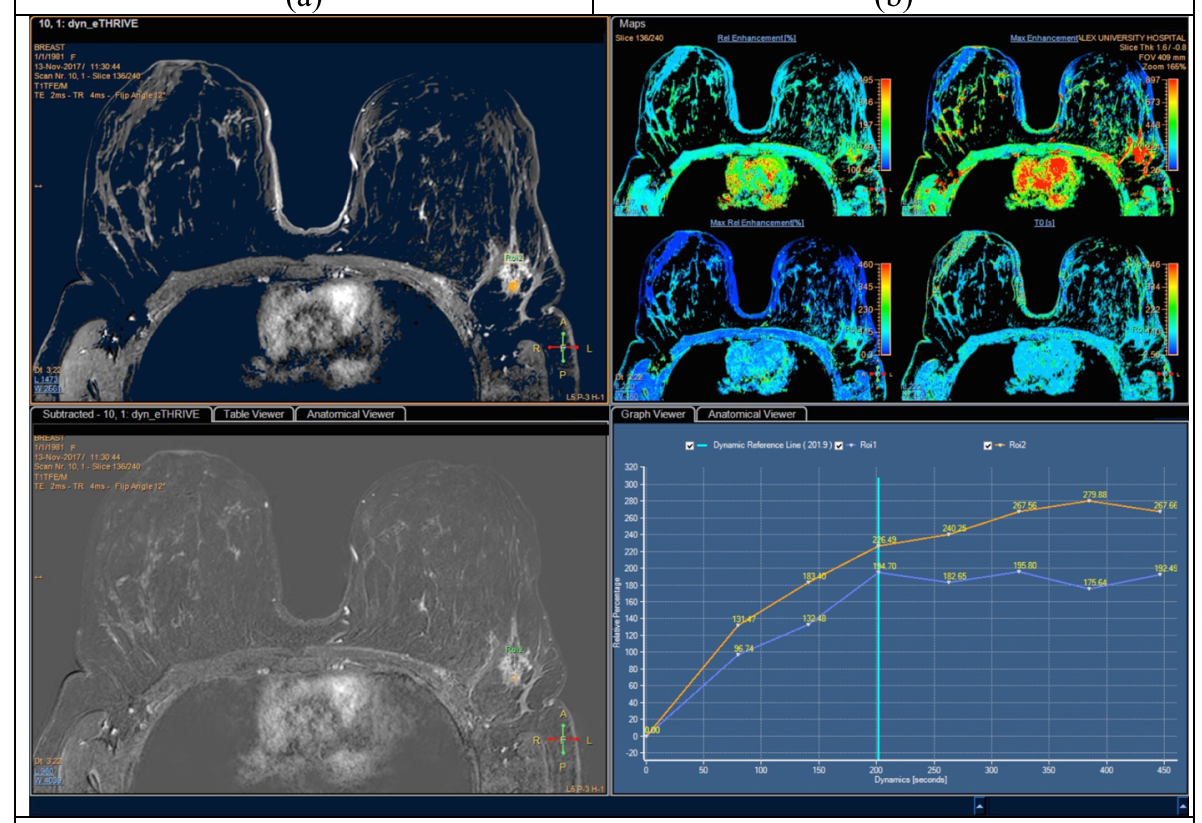

(c)

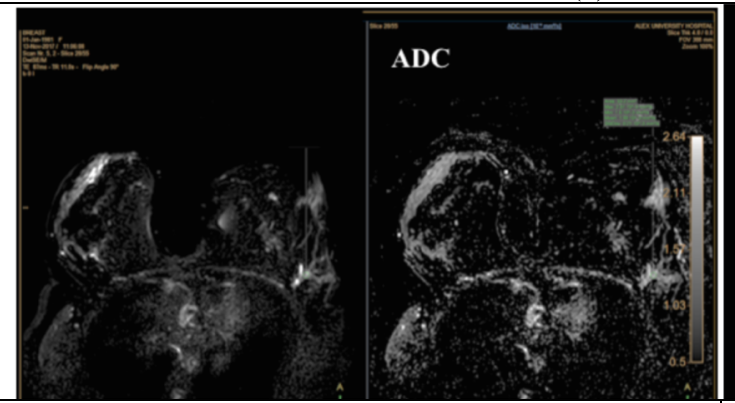

(d)

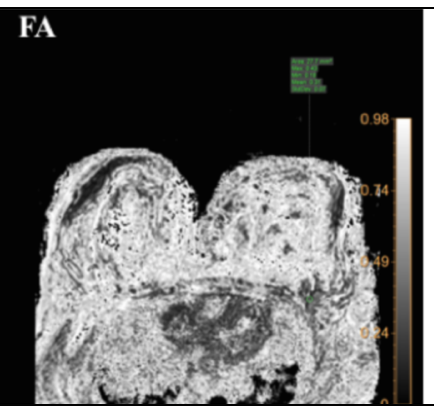

(e)

Fig. 2 40-year-old female with history of left breast cancer managed by lumpectomy on follow-up mammogram and ultrasound revealed irregular lesion seen at lumpectomy site (BIRADS 4a). CE-MRI breast $(\mathbf{a}, \mathbf{b})$ axial T1 and axial STIR showed irregular isointense lesion with central fat signal (red arrow). c Dynamic THRIVE images showed enhancing irregular lesion with suppressed central areas of fat (red arrow), showing type I curve of dynamic enhancement. $\mathbf{d}$ DWI showed no restricted diffusion ( $\left.A D C=1.9 \times 10^{-3} \mathrm{~mm}^{2} / \mathrm{s}\right)$. e FA map, FA $=0.18(<0.2)$ matching with the benignity of the lesion. Final MRI diagnosis was fat necrosis at site of lumpectomy (BIRADS 3), which was confirmed later by clinical and imaging follow-up

IBM Corp). Qualitative data were described using number and percent. Quantitative data were described using range (minimum and maximum), mean, standard deviation, and median. McNemar's Chi-square test was used for sensitivity and specificity calculation according to cut-off value which was defined using ROC curve. The significance of the obtained results was judged at the $5 \%$ level. Inter-observer agreement strength was expressed in kappa index values.

\section{Results}

The study was carried on 50 patients diagnosed by clinical examination or imaging as having breast 

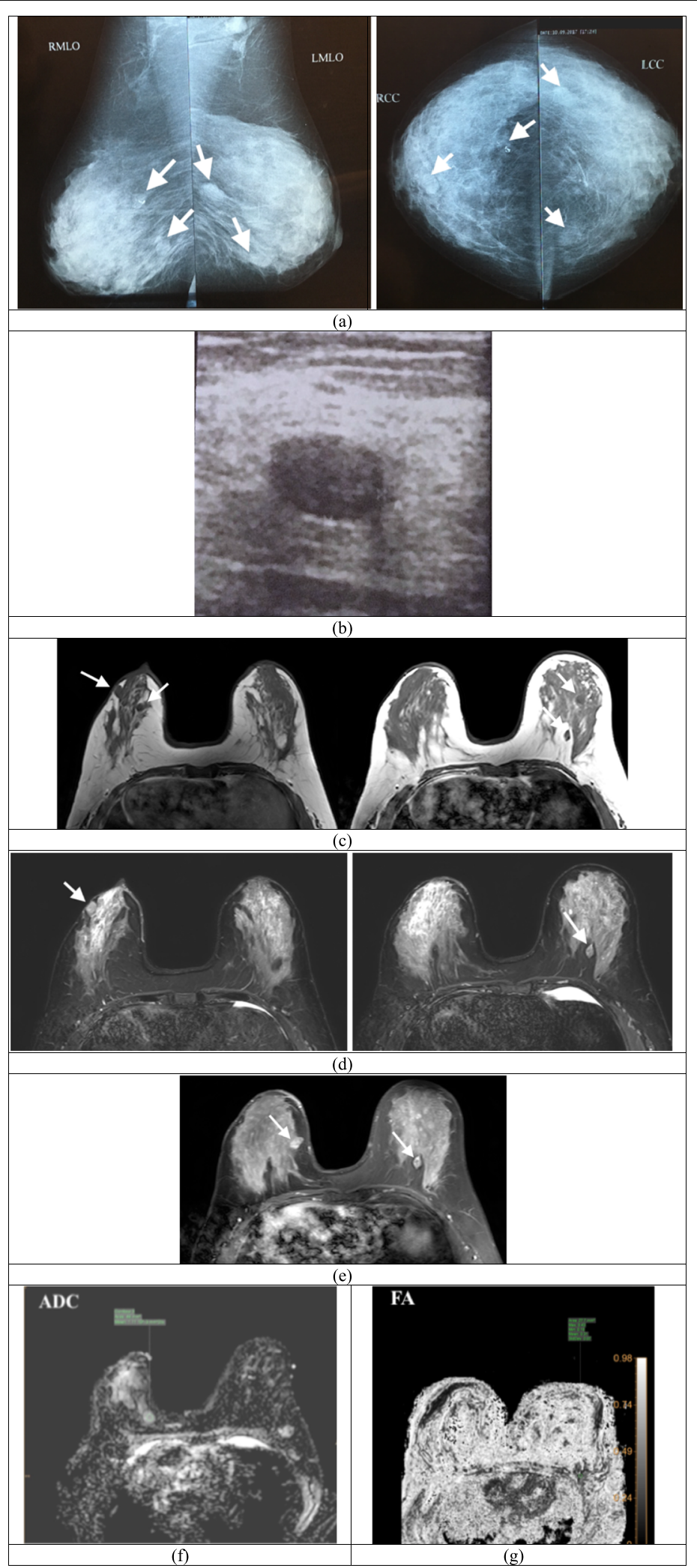

Fig. 3 (See legend on next page.) 
(See figure on previous page.)

Fig. 3 43-year-old female with positive family history presented with bilateral mastalgia. a Mammogram showed dense breast with partially obscured dense lesions at both breasts (arrows); some of them showed popcorn calcifications. b In ultrasound, they appeared as well-defined oval-shaped hypoechoic lesions, benign looking mostly fibroadenomas (BIRADS 3). CE- MRI (c, d) axial T1 and axial STIR showed bilateral welldefined oval-shaped isointense lesions (white arrows). e T1 fat saturation post contrast images showed enhancing lesions with internal dark septations. $\mathbf{f}$ No restricted diffusion ( $\left.A D C=1.1 \times 10^{-3} \mathrm{~mm}^{2} / \mathrm{s}\right)$. g FA map, FA value $=0.19(<0.2)$ matching with benignity. CE-MRI final diagnosis was benign looking lesions mostly fibroadenomas

lesion; their age ranged from 25 to 70 year-old. The most affected age group was from 45 to 65 years, 24 patients $(48 \%)$, followed by age group from 35 to 45 years (17 patients; 34\%).

Regarding the patient's clinical data, they presented with a breast lump, nipple discharge or ulceration, mastalgia, and axillary lump. Breast lump was the most common presentation; 25 case (50\%) either palpable (21 cases) or non-palpable (4 cases) and were discovered in mammogram or ultrasound.

Out of the 50 studied cases, 24 cases showed single lesion, while 26 cases showed multiple lesions; 19/26 cases showed unilateral multiple lesions (number of lesions $=48$ ) and $7 / 26$ cases showed bilateral multiple lesions (number of lesions $=19$ ).

\section{Diagnostic mammography and ultrasound breast}

Mammogram and ultrasound findings were classified according to the latest 5th edition of BIRADS (Table 1).

\section{3-T MRI breast}

The most common MRI indications in our cases were to detect any suspicious lesion in symptomatic patients especially in dense breast parenchyma or to differentiate between benign and malignant looking lesions $(28 / 50$ cases $=56 \%)$. About $17 / 50$ cases $(34 \%)$ were referred as highly suspicious for malignancy or pathologically proven malignant lesion to assess the actual size and extent of the lesion, as well as to

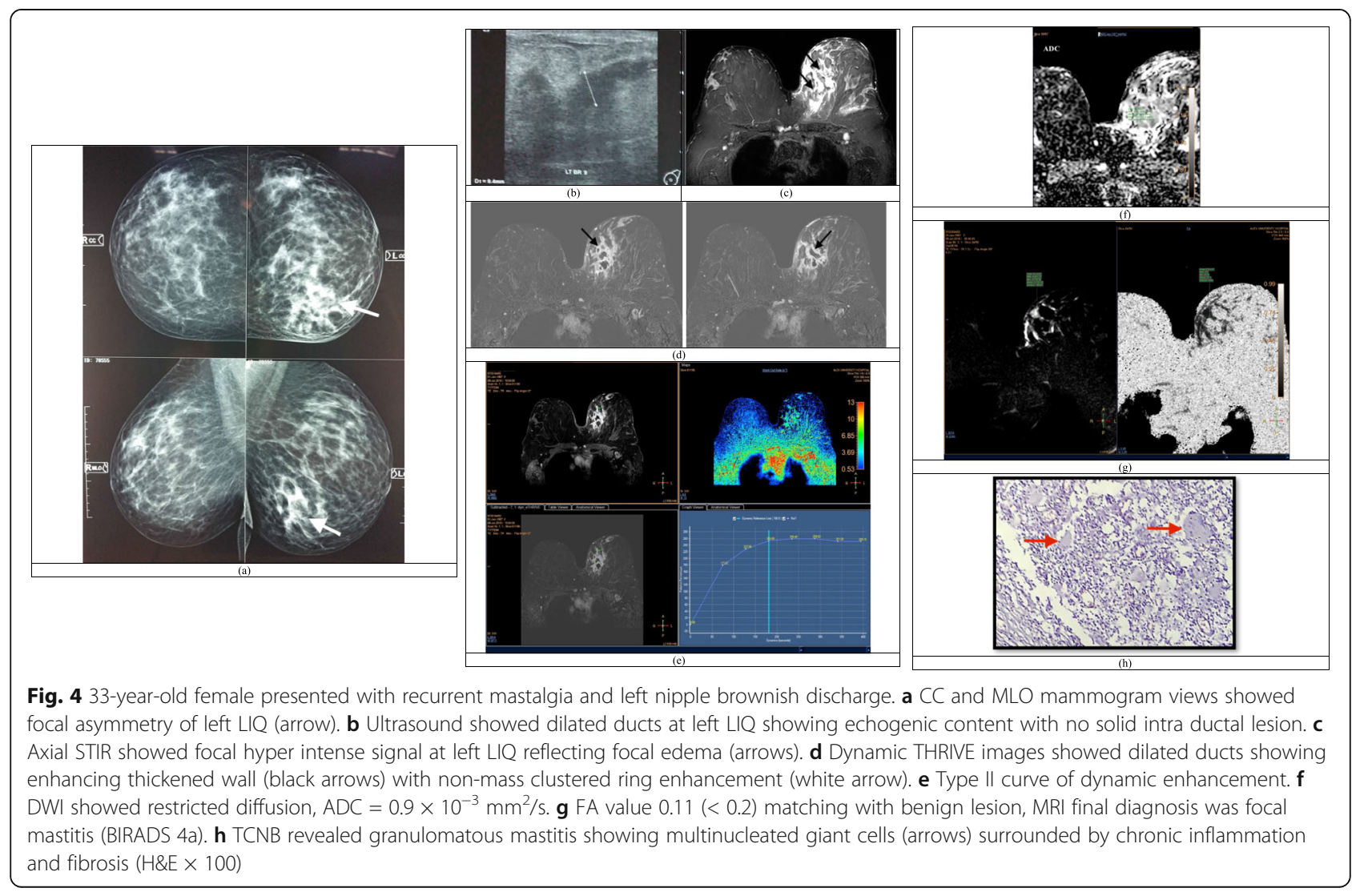




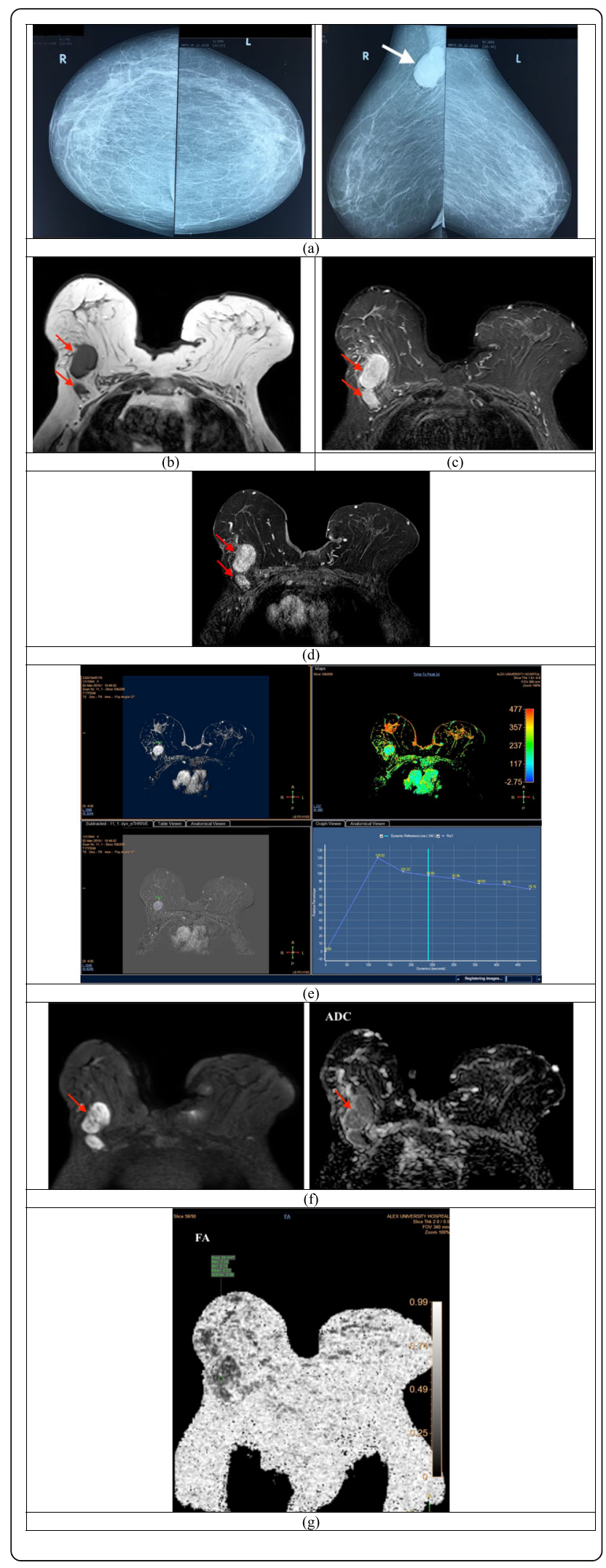

Fig. 5 70-year-old female presented with right axillary tail lump. a Mammogram, two overlapping partially circumscribed dense lesions noted at axillary tail of right breast (BIRADS 4C). CE-MRI (b) axial T1 showed oval-shaped isointense lesions with smooth margins. c Axial $\mathrm{STIR}$, the lesions showed hyper intense signal. $\mathbf{d}$ Axial dynamic THRIVE showed heterogeneous intense enhancement, with type III dynamic curve of enhancement (e). $\mathbf{f}$ DWI showed restricted diffusion (ADC $\left.=0.9 \times 10^{-3} \mathrm{~mm}^{2} / \mathrm{s}\right) . \mathbf{g}$ FA value $=0.23(>0.2)$ matching with malignant lesion. TCNB revealed mucinous carcinoma

exclude multicentricity or bilaterality in dense breast and cases of pathologically proven lobular carcinoma before starting management. The remaining 5 cases (10\%) were follow-up after lumpectomy or mastectomy.

CE-MRI breast findings of the 50 cases were classified according to the latest 5th edition of BIRADS (Table 2).

\section{Pathological assessment}

Forty-two cases out of 50 cases (84\%) were biopsied (Tables 3 and 4). The remaining 8 cases were classified as BIRADS 2 and 3 and evaluated by clinical and sonographic follow-up including fibroadenomas, fibroadenosis, and post lumpectomy fat necrosis.

\section{Correlation of DWI and DTI with the final diagnosis}

The final diagnosis of the fifty cases was based on pathology results in biopsied cases (42 cases) and on clinical and ultrasound follow-up in non-biopsied cases (8 cases). This resulted in a total number of 31 malignant cases and 19 benign cases.

Fractional anisotropy values of the detected lesions in CE-MRI breast ranged from 0.05 to 0.48 (mean $0.26 \pm 0.096 \mathrm{SD})$, while ADC values ranged from 0.5 to $2.4 \times 10^{-3} \mathrm{~mm}^{2} / \mathrm{s}$ (mean $\left.1.21 \pm 0.45 \mathrm{SD}\right)$ (Table 5) (Fig. 1).

The cut-off values in differentiation between malignant and benign lesions were $1 \times 10^{-3} \mathrm{~mm}^{2} / \mathrm{s}$ for ADC and 0.2 for FA maps using ROC curve in the analysis of ADC and FA values (Table 6).

Fractional anisotropy (FA) values and ADC values of the same lesions were correlated to the final diagnosis (Table $5)$.

In 30/50 (60\%) cases, FA values were matching with the final diagnosis; 22 malignant cases and 8 benign cases, while in $37 / 50$ cases (74\%) ADC values were matching with the final diagnosis; 20 malignant cases and 17 benign cases (Table 5).

In 30 cases out of $50(60 \%)$, FA values were matching with the pathology of the lesions as follows: 


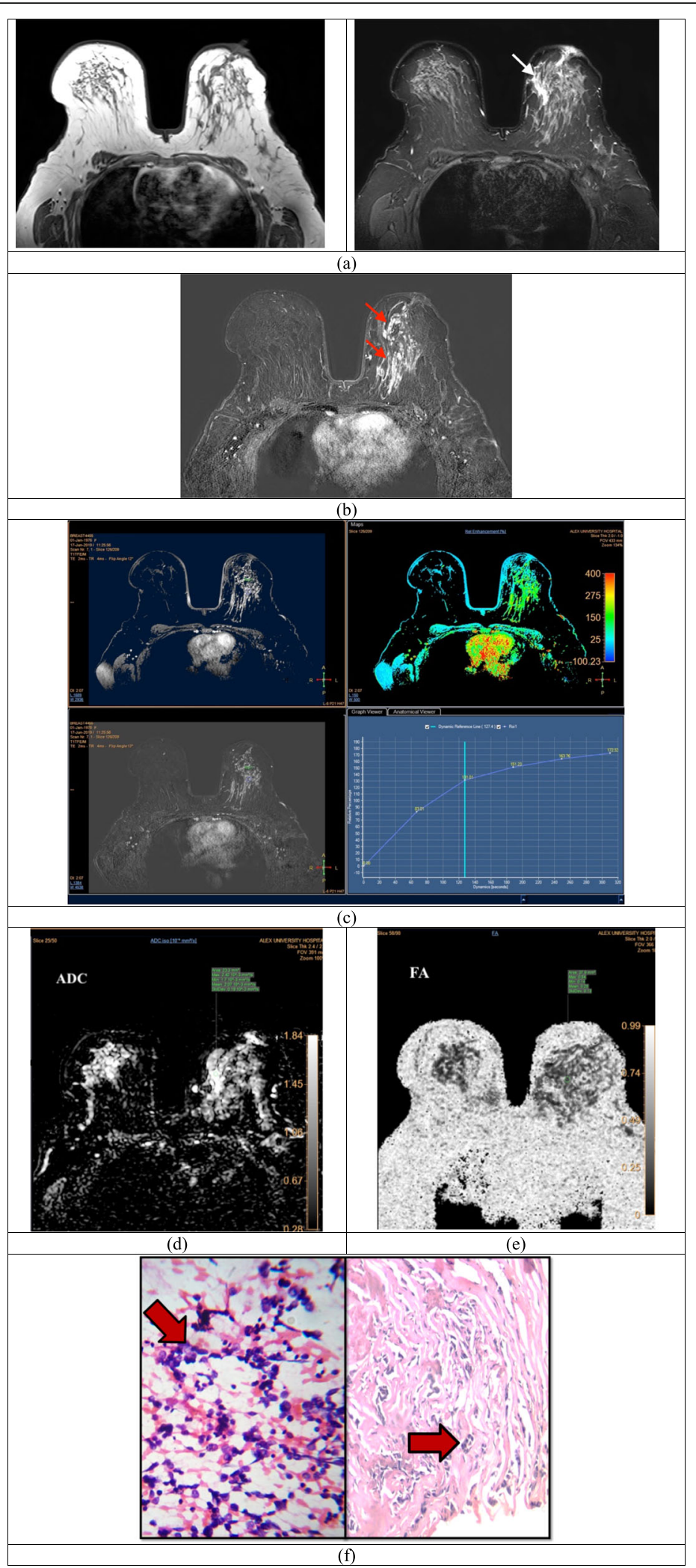

Fig. 6 (See legend on next page.) 
(See figure on previous page.)

Fig. 6 45-year-old female with history of left breast cancer managed by lumpectomy on follow-up. Mammogram showed focal asymmetry at left UIQ and ultrasound revealed dilated ducts with thickened irregular wall. CE-MRI breast (a) axial T1 (to the left) and axial STIR (to the right) showed dilated ducts at left UIQ (white arrows). b Axial contrast T1 fat saturation showed suspicious looking clumped non-mass enhancement of segmental distribution at left UIQ with no detected mass lesion. c Type I curve of dynamic enhancement. d DWI showed no restricted diffusion $\left(\mathrm{ADC}=2 \times 10^{-3} \mathrm{~mm}^{2} / \mathrm{s}\right)$. e FA map, FA $=0.28(>0.2)$ matching with malignancy. Final MRI diagnosis was suspicious non-mass enhancement (BIRADS 4c) for biopsy. $\mathbf{f}$ FNC (to the left) showed groups of malignant pleomorphic ductal cells (arrows) and a core biopsy (to the right) showed malignant glands infiltrating the stroma (arrows) $(H \& E \times 100)$. Final diagnosis was recurrent IDC

- Eight benign cases. In 7 cases out of the eight, the FA as well as the ADC values was matching with benignity. FA ranged $0.16-0.19$ and ADC ranged $1.2-1.7 \times 10^{-3} \mathrm{~mm}^{2} / \mathrm{s}$; one case was post lumpectomy fat necrosis $(\mathrm{FA}=0.18, \mathrm{ADC} 1.3 \times$ $10^{-3} \mathrm{~mm}^{2} / \mathrm{s}$ ) (Fig. 2). Another case was reactive intra mammary node (FA 0.16 , ADC $1.7 \times 10^{-3} \mathrm{~mm}^{2} / \mathrm{s}$ ); the rest of the cases were fibroadenosis and fibroadenomas $\left(\mathrm{FA}=0.19, \mathrm{ADC} 1.1 \times 10^{-3} \mathrm{~mm}^{2} / \mathrm{s}\right)$ (Fig. 3).

The remaining case was idiopathic granulomatous mastitis which showed a low $\operatorname{ADC}$ value $\left(0.9 \times 10^{-3} \mathrm{~mm}^{2} / \mathrm{s}\right)$, while FA was matching with benign condition $(\mathrm{FA}=0.11$ ) (Fig. 4).

- Twenty-two malignant cases. In 14 cases out of 22, FA and ADC values were matching with malignancy, ranged $0.25-0.48$ and $0.5-1 \times 10^{-3}$ $\mathrm{mm}^{2} / \mathrm{s}$, respectively (Fig. 5 ).

The other four cases which were pathologically proven to be ductal carcinoma in situ (DCIS) presented in MRI as suspicious linear non-mass enhancement; ADC values were misleading more with benignity, ranged $1.2-1.8 \times 10^{-3} \mathrm{~mm}^{2} / \mathrm{s}$, while FA values ranged $0.28-0.35$ matching with malignant lesion (Fig. 6).

Three cases were infiltrating ductal carcinoma (IDC), presented as a mass lesion; ADC values were more with benignity ranged $1.2-1.8 \times 10^{-3} \mathrm{~mm}^{2} / \mathrm{s}$, while FA values were 0.25-0.35 matching with malignant lesion (Figs. 7 and 8), and one case was invasive lobular carcinoma in which the ADC value were more with benignity $\left(1.2 \times 10^{-3} \mathrm{~mm}^{2} / \mathrm{s}\right)$, while FA value was 0.39 matching with malignant lesion.

Regardless of the correlation with the final pathology of the lesions, there was agreement between the results of ADC and FA in 33 cases out of 50 (66\%), 19 cases malignant and 14 benign cases.

Generally, in malignant cases which were presented in CE-MRI as mass associated with suspicious non-mass enhancement, higher FA and lower $\mathrm{ADC}$ values were detected within the mass than the
FA and ADC values of the associated non-mass enhancement, respectively.

By analyzing the data of the 50 cases, it was found that the sensitivity and specificity of DWI in differentiation between benign and malignant lesions about $90 \%$ and 63\%, respectively, with PPV 90\% and NPV 62\% with Pvalue $\leq 0.05$. While the DTI showed lower sensitivity and specificity about $81 \%$ and $51.7 \%$, respectively, with PPV $78.9 \%$ and NPV $54.8 \%$ with $P$-value $\leq 0.05$ (Table 6) (Fig. 8).

Although the study has showed that DTI has lower sensitivity and specificity in comparison to DWI, yet in 30 cases out of 50 (60\% of the cases), the final diagnosis of the detected lesions in $3-\mathrm{T}$ DCE-MRI showed matching results with FA values, which may indicate that adding DTI to conventional breast MRI may improve the ability to distinguish between benign and malignant lesions in some indeterminate cases.

\section{Discussion}

The role of dynamic contrast-enhanced magnetic resonance imaging (DCE-MRI) in local staging and breast lesion characterization is well established [3].

Compared with 1.5-T MRI, 3.0-T imaging allows higher signal-to-noise ratio, improved spatial resolution, and faster scanning and therefore may be able to test advanced added techniques as DTI to help in predicting the malignancy of lesions more accurately [21].

Based on the fact that DTI is a diffusion imaging applied in different directions, in trial to assess the diagnostic role of DTI, we compared it to DWI as a well-established diffusion parameter known for its good sensitivity and specificity in characterization of breast lesions and currently is an integral part of DCE-MRI breast.

This study included 50 patients presented with breast lesion, 31 malignant cases and 19 benign cases; their age ranged from 25 to 70 years, the most common age group (45-65 years) about $48 \%$ of the cases. 


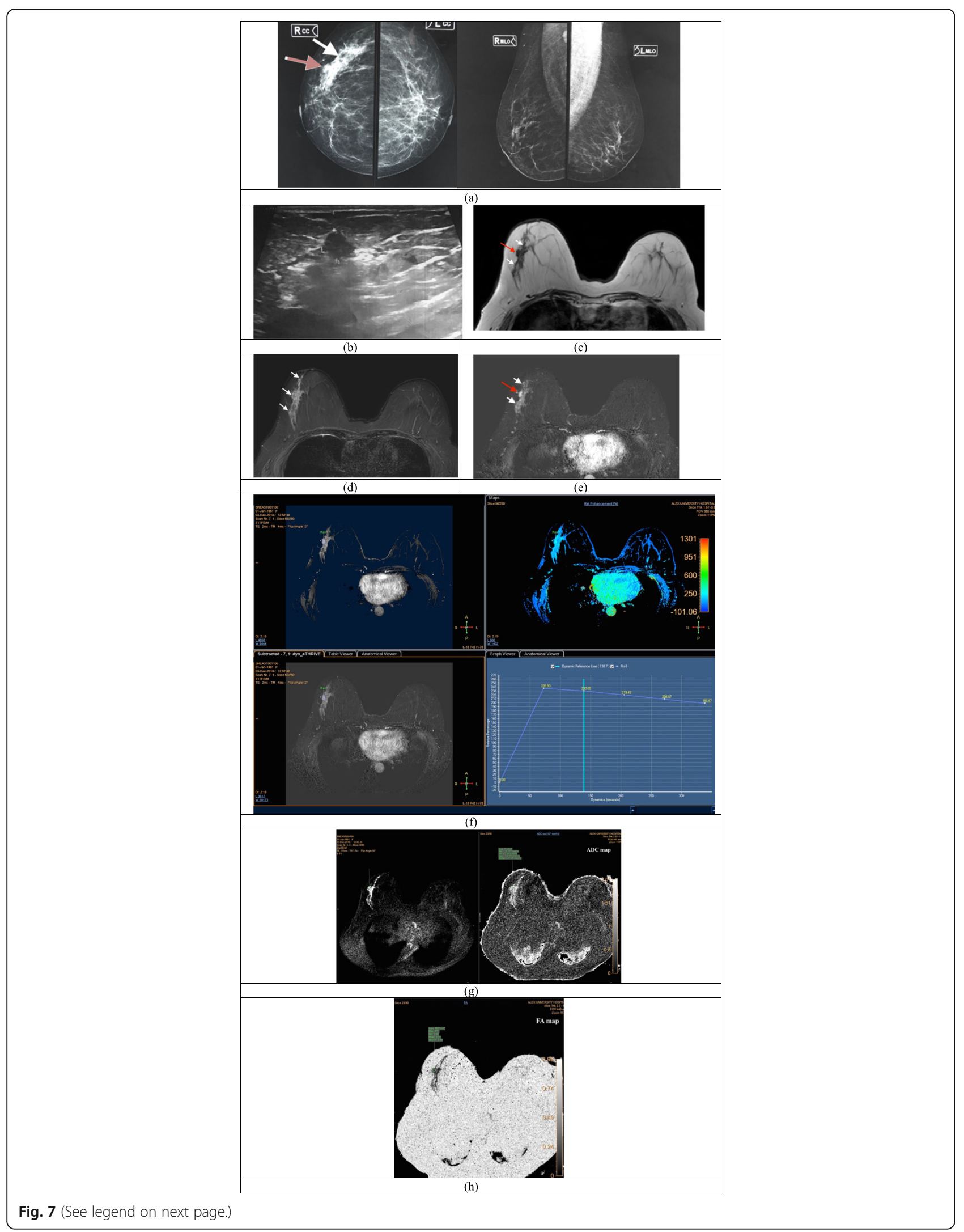


(See figure on previous page.)

Fig. 7 57-year-old female presented by right uniparous spontaneous bloody discharge. a Mammogram showed one view asymmetry at outer part of right breast in CC view (white arrow) and partially obscured dense lesion (red arrow). b Ultrasound showed hypoechoic lesion with irregular margins (red arrow) and related dilated duct (white arrow) (BIRADS 5), referred for MRI to assess extent of the lesion. c Axial T1 showed irregular hypointense lesion (red arrow). d Axial STIR showed focal linear hyperintense signal (white arrows). e Axial dynamic THRIVE images showed irregular enhancing lesion (red arrow) previously seen in mammogram and ultrasound, associated with related suspicious linear non-mass enhancement matching with the linear focal asymmetry seen in CC view mammogram. (white arrows). f Type III curve of dynamic enhancement. $\mathbf{g}$ DWI showed no diffusion restriction, $\mathrm{ADC}=1.4 \times 10^{-3} \mathrm{~mm}^{2} / \mathrm{s}$. h FA $=0.24(>0.2)$ matching with malignancy. TCNB revealed IDC

Seven cases aged 25 to $<40$ years were included in this MRI study. Although they were classified by ultrasound as BIRADS 1, 2, or 3, they were assessed by MRI for clinical purposes to detect any suspicious lesion in symptomatic patients with BIRADS 1 or 2 , especially in dense breast parenchyma or positive family history and to add MRI assessment for BIRADS 3 lesions, as well as for research purpose aiming to include benign lesions for testing DTI in addition to the malignant lesions.

As a result, the study included good spectrum of benign lesions as fibroadenosis, fibroadenomas, duct papillomatosis, granulomatous mastitis, and lymphocytic mastitis in addition to different pathological subtypes of malignant lesions as DCIS, IDC, invasive lobular carcinoma, Paget's disease of the nipple, and mucinous carcinoma.

Although usage of DWI as a part of CE-MRI breast has been established with low ADC value as a feature of breast malignancy, there are contradicting results regarding the added value of DTI in differentiating between malignant and benign lesions. FA is the most studied DTI parameter. Some studies have reported higher FA in malignant lesions compared with benign ones as Teruel et al. [22], Baltzer et al. [4], and Tsougos et al. [23], while others like Partridge et al. [15], Eyal et al. [24], and Cakir et al. [14] have found no significant difference.
Our first concern was to optimize the DTI sequence to obtain good quality images for accurate reading of $\mathrm{FA}$ values.

For this aim, DTI sequence in our study was tested in a 3-T MRI machine which has been shown in prior studies improved diagnostic performance compared to $1.5-\mathrm{T}$ due to superior signalto-noise ratio (SNR) and increased spatial resolution [25].

Also, most of the published previous studies were testing DTI in breast imaging using a 1.5-T MRI machine (for example [4, 26]), so it was beneficial to provide some data about DTI in 3-T MRI.

Regarding the DTI sequence, medium number of gradient diffusion directions was chosen to ensure good quality of the sequence and at same time accepted acquisition time for the sequence. In addition, the DTI sequence was taken before contrast administration which may be preferable to avoid any possible confounding effect of the contrast on DTI parameters.

While comparing the DTI to DWI in our study, the DTI achieved lower sensitivity and specificity than DWI, it showed about $81 \%$ sensitivity and $51.7 \%$ specificity with cut-off value of FA to differentiation between malignant and benign lesions (0.2), compared to $90 \%$ sensitivity and $63 \%$ specificity of DWI. This is similar to results of a study,
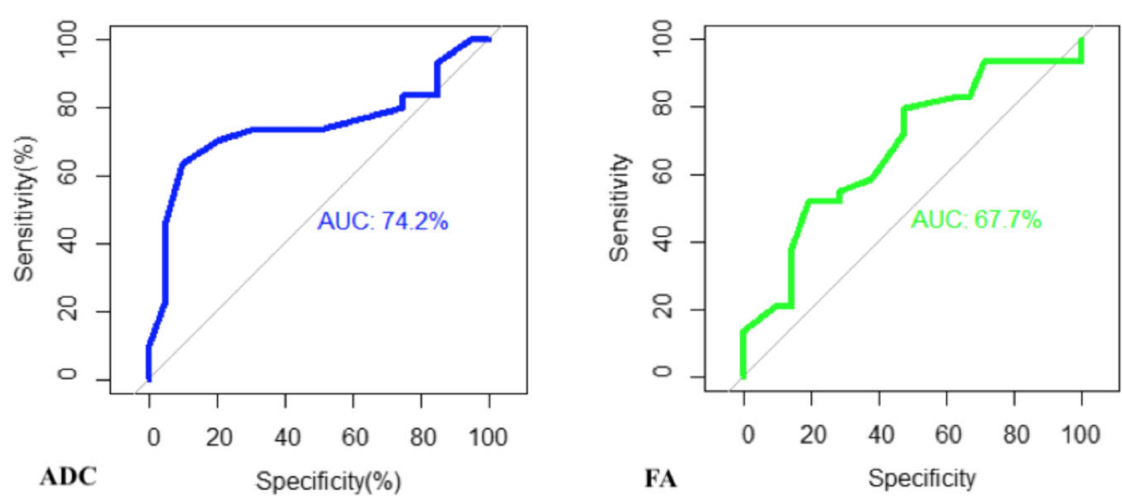

Fig. 8 ROC curve of DWI "ADC" (to the left) and DIT "FA" (to the right) to predict breast malignant tumor 
Baltzer et al. [4], which was carried on 59 patients to test diffusion tensor imaging of 1.5-T MRI breast revealed sensitivity $79.6 \%$ and specificity $64.7 \%$ with cut-off value of FA (0.19).

Jiang et al. [26] studied 88 patients with 88 breast lesions who underwent DTI on 1.5-T MRI reported cut-off values of ADC and FA for malignant breast lesions $\left(1 \pm 0.33 \times 10^{-3} \mathrm{~mm}^{2} / \mathrm{s}\right.$ and $\left.0.2 \pm 0.05\right)$, respectively, similar to the cut-off values in our study $\left(1 \times 10^{-3} \mathrm{~mm}^{2} / \mathrm{s}\right.$ and 0.2$)$, respectively.

Although DWI showed better overall sensitivity and specificity than DTI, DTI showed conclusive results in cases with DCIS, in which the DWI showed false positive or false negative results. This may indicate that FA can be more sensitive to changes in the microstructure of the cells before increasing cellularity of the lesion.

On the other hand, unlike our study, Cakrik et al. [14], while examining DWI and DTI in 55 breast lesions (30 malignant, 25 benign) using 3-T MRI, concluded that ADC of DWI was discriminative for benign and malignant breast lesions while FA measurement was not. These controversies are encouraging for more investigations about optimization of DTI and testing its added value in MRI breast imaging.

Also, it was noticed that FA expressed better results in malignant lesions rather than benign lesions. This can be attributed to larger number of included patients older than 40 years old with higher number of malignant lesions than younger patients with benign tumors. Further studies with more number of benign lesions are advised to ensure that there is no bias in our analysis due to age factor.

There were some limitations in our study; the study was carried on a relatively small number of patients; however, a statistically significant difference was found suggesting that further prospective studies with a larger number of cases would be beneficial.

Detailed lesion assessment in DTI was limited by technical issues related to the single-shot echo-planar imaging (EPI) technique due to its limited spatial resolution and its frequent artifacts. The results of recent multicenter breast DWI trials stated that about 13 to $29 \%$ of cases are being excluded for DWI technical issues [27-29].

Also, the DTI sequence was time-consuming (the sequence time for 15 directions was about $10 \mathrm{~min}$ ) with subsequent prolonged time of the whole MRI study to about $25-35 \mathrm{~min}$. This time was not tolerated by all patients especially obese or asthmatic patients.

As a final limitation, ROIs were manually defined on DTI maps for each exam after comparing DCE-MRI and
DTI. Manual ROI is prone to operator dependence and sampling error, especially for irregularly shaped masses or non-mass enhancement.

\section{Conclusion}

DTI can be used as an adjuvant sequence in diagnostic CE-MRI breast. While the DWI is still the most established diffusion parameter for differentiation between benign and malignant breast lesions, DTI may be helpful in further characterization of tumor microstructure which definitely needs further investigations.

\section{Abbreviations}

DCE-MRI: Dynamic contrast-enhanced MRI; DTI: Diffusion tensor imaging; DWI: Diffusion-weighted imaging; ADC: Apparent diffusion coefficient;

BOLD: Blood-oxygen-level-dependent; STIR: Short T1 inversion recovery; sSEPI: Single-shot spin echo planner imaging; SPAIR: Spectral attenuated inversion recovery; FA: Fraction anisotropy; ROI: Region of interest;

ROC: Receiver operating characteristic; BIRADS: Breast Imaging and Reporting System; TCNB: Tru cut needle biopsy; IDC: Infiltrating ductal carcinoma;

DCIS: Ductal carcinoma in situ

\section{Acknowledgements}

Not applicable

\section{Authors' contributions}

DA: design of the study, data collection, MR image interpretation, statistical analysis, and manuscript writing. AA: performed histopathological revision of the cases. AH and AEA: manuscript editing and revision sequence alignment. $\mathrm{HH}$ : interpretation of MR images, manuscript editing, and sequence alignment. All authors approved the final manuscript.

\section{Funding}

No sources of funding (not applicable).

\section{Availability of data and materials}

The datasets used and/or analyzed during the current study are available from the corresponding author on reasonable request.

\section{Declarations}

Consent for publications

Not applicable.

\section{Ethics approval and consent to participate}

This study was approved by the Research Ethics Committee of the Faculty of Medicine at Alexandria University in Egypt on 2018 (reference number is 0201092, IRB number 00012098). Written informed consent was obtained from all patients included in this study.

\section{Competing interests}

The authors declare that they have no competing interests.

\section{Author details}

${ }^{1}$ Department of Diagnostic and Interventional Radiology, Faculty of Medicine, Alexandria University, Alexandria, Egypt. ${ }^{2}$ Department of Diagnostic and Interventional Radiology, Faculty of Medicine, Alexandria University Hospital, Champollion Street, Al-Azarita, Alexandria 21526, Egypt.

${ }^{3}$ Department of Pathology, Faculty of Medicine, Alexandria University, Alexandria, Egypt.

Received: 18 January 2021 Accepted: 23 March 2021

Published online: 07 April 2021

References

1. DeSantis CE, Ma J, Sauer AG, Newman LA, Jemal A (2017) Breast cancer statistics, 2017, racial disparity in mortality by state. A Cancer J Clin. 67(6): 439-448. https://doi.org/10.3322/caac.21412 
2. Kul S, Cansu A, Alhan E, Dinc H, Gunes G, Reis A (2011) Contribution of diffusion-weighted imaging to dynamic contrast-enhanced MRI in the characterization of breast tumors. AJR 196(1):210-217. https://doi.org/1 0.2214/AJR.10.4258

3. Perry N, Broeders M, de Wolf C, Törnberg S, Holland R, von Karsa L (2008) European quidelines for quality assurance in breast cancer screening and diagnosis. Fourth edition--summary document. Ann Oncol. 19(4):614-622. https://doi.org/10.1093/annonc/mdm481

4. Baltzer PAT, Schäfer A, Dietzel M, Grässel D, Gajda M, Camara O, Kaiser WA (2011) Diffusion tensor magnetic resonance imaging of the breast: a pilot study. Eur Radiol. 21(1):1-10. https://doi.org/10.1007/s00330-010-1901-9

5. Peters NHGM, Borel Rinkes IHM, Zuithoff NPA, Mali WPTM, Moons KGM, Peeters PHM (2008) Meta-analysis of MR imaging in the diagnosis of breast lesions. Radiology. 246(1):116-124. https://doi.org/10.1148/radiol.2461061298

6. Sardanelli F, Giuseppetti GM, Panizza P, Bazzocchi M, Fausto A, Simonetti G, Lattanzio V, del Maschio A (2004) Sensitivity of MRI versus mammography for detecting foci of multifocal, multicentric breast cancer in fatty and dense breasts using the whole-breast pathologic examination as a gold standard. AJR Am J Roentgenol. 183(4):1149-1157. https://doi.org/10.2214/a jr.183.4.1831149

7. Wiederer J, Pazahr S, Leo C, Nanz D, Boss A (2014) Quantitative breast MRI: 2D histogram analysis of diffusion tensor parameters in normal tissue. Magn Reson Mater Phy. 27(2):185-193. https://doi.org/10.1007/s10334-013-0400-9

8. Partridge SC, Nissan N, Rahbar H, Kitsch AE, Sigmund EE (2017) Diffusionweighted breast MRI: clinical applications and emerging techniques. J Magn Reson Imaging. 45(2):337-355. https://doi.org/10.1002/jmri.25479

9. Kuhl CK, Jost P, Morakkabati N, Zivanovic O, Schild HH, Gieseke J (2006) Contrast-enhanced MR imaging of the breast at 3.0 and $1.5 \mathrm{~T}$ in the same patients: Initial Experience. Radiology 239(3):666-676

10. Rakow-Penner R, Hargreaves B, Glover G, Daniel B (2009) Breast MRI at 3T. Applied radiology.

11. Chang RF, Chen HH, Chang YC, Huang CS, Chen JH, Lo CM (2016) Quantification of breast tumor heterogeneity for ER status, HER2 status, and TN molecular subtype evaluation on DCE-MRI. Magn Reson Imaging. 34(6): 809-819. https://doi.org/10.1016/j.mri.2016.03.001

12. Parsian $\mathrm{S}$, Rahbar $\mathrm{H}$, Allison $\mathrm{KH}$, Demartini WB, Olson ML, Lehman CD et al (2012) Nonmalignant breast lesions: ADCs of benign and high-risk subtypes assessed as false-positive at dynamic enhanced MR imaging. Radiology. 265(3):696-706. https://doi.org/10.1148/radiol.12112672

13. Basser PJ, Mattiello J, LeBihan D (1994) MR diffusion tensor spectroscopy and imaging. Biophys J. 66(1):259-267. https://doi.org/10.1016/S0006-34 95(94)80775-1

14. Cakir O, Arslan A, Inan N, Anık Y, Sarısoy T, Gumustas S, Akansel G (2013) Comparison of the diagnostic performances of diffusion parameters in diffusion weighted imaging and diffusion tensor imaging of breast lesions. Eur J Radiol. 82(12):e801-e806. https://doi.org/10.1016/j.ejrad.2013.09.001

15. Partridge SC, Ziadloo A, Murthy R, White SW, Peacock S, Eby PR, DeMartini WB, Lehman CD (2010) Diffusion tensor MRI: preliminary anisotropy measures and mapping of breast tumors. J Magn Reson Imag. 31(2):339347. https://doi.org/10.1002/jmri.22045

16. Ozal ST, Inci E (2018) Diagnostic values of diffusion tensor parameters in $3 \mathrm{~T}$ breast magnetic resonance imaging: differentiation of breast tumors from bilateral healthy breast parenchyma. Niger J Clin Pract. 21(11):1531-1536

17. Ohtake T, Kimijima I, Fukushima T, Yasuda M, Sekikawa K, Takenoshita S et al (2001) Computer-assisted complete three-dimensional reconstruction of the mammary ductal/lobular systems: implications of ductal anastomoses for breast-conserving surgery. Cancer 91(12):2263-2272

18. Going JJ, Moffat DF (2004) Escaping from Flatland: clinical and biological aspects of human mammary duct anatomy in three dimensions. J Pathol. 203(1):538-544. https://doi.org/10.1002/path.1556

19. Hancu I, Govenkar A, Lenkinski RE, Lee S-K (2013) On shimming approaches in 3T breast MRI. Magn Reson Med 69(3):862-867

20. Plaza MJ, Morris EA, Thakur SB (2016) Diffusion tensor imaging in the normal breast: influences of fibroglandular tissue composition and background parenchymal enhancement. Clin Imaging. 40(3):506-511. https://doi.org/10.1016/j.clinimag.2015.12.001

21. Arponent $O$, Sudah M, Masarwah A, Taina M, Rautiainen S, Könönen M et al (2015) Diffusion-weighted imaging in 3.0 Tesla breast MRl: diagnostic performance and tumor characterization using small subregions vs. whole tumor regions of interest. PLOS ONE 10(10):e0141833
22. Teruel JR, Goa PE, Sjøbakk TE, Østlie A, Fjøsne HE, Bathen TF (2016) Diffusion weighted imaging for the differentiation of breast tumors: from apparent diffusion coefficient to high order diffusion tensor imaging. J Magn Reson Imaging. 43(5):1111-21. https://doi.org/10.1002/jmri.25067.

23. Tsougos I, Svolos P, Kousi E, Athanassiou E, Theodorou K, Arvanitis D, Fezoulidis I, Vassiou K (2014) The contribution of diffusion tensor imaging and magnetic resonance spectroscopy for the differentiation of breast lesions at 3T. Acta Radiol. 55(1):14-23. https://doi.org/10.1177/02841851134 92152

24. Eyal E, Shapiro-Feinberg M, Furman-Haran E, Grobgeld D, Golan T, Itzchak Y, Catane R, Papa M, Degani H (2012) Parametric diffusion tensor imaging of the breast. Investig Radiol. 47(5):284-291. https://doi.org/10.1097/RLI.0b013 e3182438e5d

25. Spick C, Szolar DHM, Preidler KW, Reittner P, Rauch K, Brader P, Tillich M, Baltzer PA (2018) 3 Tesla breast MR imaging as a problem-solving tool: diagnostic performance and incidental lesions. PLOS ONE 13(1):e0190287. https://doi.org/10.1371/journal.pone.0190287

26. Jiang R, Ma Z, Dong H, Sun S, Zeng X, Li X (2016) Diffusion tensor imaging of breast lesions: evaluation of apparent diffusion coefficient and fractional anisotropy and tissue cellularity. Br J Radiol. 89(1064):20160076. https://doi. org/10.1259/bjr.20160076

27. Rahbar H, Zhang Z, Chenevert TL, Romanoff J, Kitsch A, Hanna LG et al (2019) Utility of diffusion weighted imaging to decrease unnecessary biopsies prompted by breast MRI: a trial of the ECOG-ACRIN Cancer Research Group (A6702). Clin Cancer Res. 25(6):1756-1765. https://doi.org/1 0.1158/1078-0432.CCR-18-2967

28. Newitt DC, Zhang Z, Gibbs JE, Partridge SC, Chenevert TL, Rosen MA, Bolan PJ, Marques HS, Aliu S, Li W, Cimino L, Joe BN, Umphrey H, Ojeda-Fournier H, Dogan B, Oh K, Abe H, Drukteinis J, Esserman LJ, Hylton NM, for the ACRI N Trial Team and I-SPY 2 TRIAL Investigators (2019) Test-retest repeatability and reproducibility of ADC measures by breast DWI: results from the ACRIN 6698 trial. J Magn Reson Imaging. 49(6):1617-1628. https://doi.org/10.1002/ jmri.26539

29. Artridge SC, Zhang Z, Newitt DC, Gibbs JE, Chenevert TL, Rosen MA et al (2018) Diffusion-weighted MRI findings predict pathologic response in neoadjuvant treatment of breast cancer: the ACRIN 6698 Multicenter Trial. Radiology. 289(3):618-627. https://doi.org/10.1148/radiol.2018180273

\section{Publisher's Note}

Springer Nature remains neutral with regard to jurisdictional claims in published maps and institutional affiliations.

\section{Submit your manuscript to a SpringerOpen ${ }^{\circ}$ journal and benefit from:}

- Convenient online submission

- Rigorous peer review

- Open access: articles freely available online

- High visibility within the field

- Retaining the copyright to your article

Submit your next manuscript at $>$ springeropen.com 